hep-ph/0703044

YITP-07-12

KUNS-2065

\title{
Relaxed fine-tuning in models with non-universal gaugino masses
}

\author{
Hiroyuki Abe ${ }^{1, *}$ Tatsuo Kobayashi ${ }^{2, \dagger}$ and Yuji Omura ${ }^{3, \ddagger}$ \\ ${ }^{1}$ Yukawa Institute for Theoretical Physics, Kyoto University, Kyoto 606-8502, Japan \\ ${ }^{2}$ Department of Physics, Kyoto University, Kyoto 606-8502, Japan \\ ${ }^{3}$ Department of Physics, Kyoto University, Kyoto 606-8501, Japan
}

\begin{abstract}
We study, in a bottom-up approach, the fine-tuning problem between soft SUSY breaking parameters and the $\mu$-term for the successful electroweak symmetry breaking in the minimal supersymmetric standard model. It is shown that certain nontrivial ratios between gaugino masses, that is non-universal gaugino masses, are necessary at the GUT scale, in order for the fine-tuning to be reduced above $10 \%$ order. In addition, when all the gaugino masses should be regarded as independent ones in their origins, a small gluino mass $M_{3} \lesssim 120 \mathrm{GeV}$ and a non-vanishing $A$-term $A_{t} \sim O\left(M_{3}\right)$ associated to top squarks are also required at the GUT scale as well as the non-universality. On the other hand, when we consider some UV theory, which fixes ratios of soft SUSY breaking parameters as certain values with the overall magnitude, heavier spectra are allowed. It is favored that the gluino and wino masses are almost degenerate at the weak scale, while wider region of bino mass is favorable.
\end{abstract}

\footnotetext{
*E-mail address: abe@yukawa.kyoto-u.ac.jp

${ }^{\dagger}$ E-mail address: kobayash@gauge.scphys.kyoto-u.ac.jp

${ }^{\ddagger}$ E-mail address: omura@scphys.kyoto-u.ac.jp
} 


\section{Introduction}

Supersymmetric extension of the standard model (SM) is one of the most promising candidates for a new physics at the TeV scale. It can stabilize the huge hierarchy between the electroweak (EW) scale and the Planck scale. In particular, the minimal supersymmetric standard model (MSSM) is interesting from the viewpoint of its minimality. Also the MSSM unifies three gauge couplings of SM gauge interactions at the grand unified theory (GUT) scale $M_{G U T} \sim 2 \times 10^{16}$ more precisely. Furthermore, supersymmetric standard models provide sources for the dark matter.

Among such attractive features, the most remarkable one would be the radiative EW symmetry breaking [1]. The MSSM can automatically break EW symmetry due to the large logarithmic correction to the soft supersymmetry (SUSY) breaking mass $m_{H_{u}}$ for the up-sector Higgs field [2],

$$
\Delta m_{H_{u}}^{2} \sim-\frac{3 y_{t}^{2}}{4 \pi^{2}} m_{\tilde{t}}^{2} \ln \frac{\Lambda}{m_{\tilde{t}}},
$$

which determines the size of $Z$-boson mass $M_{Z}$ as

$$
\frac{1}{2} M_{Z}^{2} \sim-\mu^{2}-m_{H_{u}}^{2}
$$

through a minimization condition for the Higgs potential. Here, $y_{t}$ is the top Yukawa coupling, $m_{\tilde{t}}$ is the top squark mass, $\Lambda$ is the cut-off scale, and $\mu$ is the SUSY mass of up- and down-sector Higgs fields. We have assumed a (moderately) large value of $\tan \beta=\left\langle H_{u}\right\rangle /\left\langle H_{d}\right\rangle$.

On the other hand, the MSSM predicts the lightest CP-even Higgs mass at one-loop level,

$$
m_{h}^{2} \leq M_{z}^{2}+\frac{3 m_{t}^{4}}{4 \pi^{2} v^{2}} \ln \frac{m_{\tilde{t}^{2}}}{m_{t}^{2}}+\cdots
$$

The experimental bound $m_{h} \geq 114.4 \mathrm{GeV}$ requires $m_{\tilde{t}} \gtrsim 500 \mathrm{GeV}$. This value of $m_{\tilde{t}}$ leads to quite large correction $\Delta m_{H_{u}}^{2}$. Thus, to obtain $M_{Z}$, we need typically a few percent fine-tuning between the SUSY mass $\mu$ and the soft SUSY breaking mass $m_{H_{u}}$ at the GUT scale, which are not related to each other in general. This is sometimes called a 'little hierarchy problem' 3]. There have been several works recently addressing this issue [4]-[15]. Most of them, however, are based on some specific models.

Here, we study the fine-tuning problem from the bottom-up viewpoint, and show what kind of model can relax this sort of fine-tuning. We will take two kinds of stances. One is a complete bottom-up approach, where all the soft SUSY breaking parameters are considered as independent ones to each other in their origins. In this case, we have to care about the sensitivity of the EW scale ( $Z$-boson mass) to all the parameters at the GUT scale. The other is, in a sense, a half top-down approach. We suppose some ultra-violet (UV) theories which fix certain ratios between the soft parameters at the GUT scale. Then we consider the fine-tuning between the remaining independent ones. We will show preferable values of the ratios between gaugino masses and the $A$-term. 
Indeed, several models lead to non-universal gaugino masses as well as non-universal scalar masses and A-terms, e.g. moduli mediation [16], anomaly mediation [17], mirage mediation [18, 9] and the SUSY breaking scenario, where F-components of gauge nonsinglets are dominant [15, 19]. (See also Ref. [20] for several classes of models leading to non-universal gaugino masses with certain ratios.) Scalar masses and $A$-terms are more model-dependent. However, in each model, ratios of gaugino masses and scalar masses as well as $A$-terms are fixed as certain values. In these models, the independent parameter for SUSY breaking terms corresponds to the overall magnitude of SUSY breaking, say $M$, and we should concentrate to only the fine-tuning of the overall magnitude $M$.

The sections of this paper are organized as follows. In Section 2 , we briefly review the fine-tuning problem in the MSSM, and introduce fine-tuning parameters. In Section 3, we discuss how the fine-tuning can be reduced when all the soft SUSY breaking parameters are regarded as independent ones. In Section 4, on the other hand, we examine the fine-tuning problem under the assumption that certain ratios between soft parameters, especially between gaugino masses, are fixed by some UV theories and find preferable ratios which reduce the fine-tuning. Section 5 is devoted to conclusions and discussions.

\section{Fine-tuning problem in MSSM}

In this section we review the fine-tuning problem in the MSSM shortly, and then introduce fine-tuning parameters describing the sensitivity of the EW sale to the soft parameters at the GUT scale.

The MSSM Higgs sector is described by the superpotential,

$$
W_{S U S Y}=\mu H_{u} H_{d}+y_{t} Q_{3} U_{3} H_{u}
$$

and the relevant soft SUSY breaking terms are written as,

$$
V_{\text {solf }}=m_{H u}^{2}\left|H_{u}\right|^{2}+m_{H d}^{2}\left|H_{d}\right|^{2}+m_{Q_{3}}^{2}+m_{U_{3}}^{2}+\left(\mu B H_{u} H_{d}+y_{t} A_{t} Q_{3} U_{3} H_{u}+\text { h.c. }\right),
$$

where $m_{H_{d}}, m_{Q_{3}}$ and $m_{U_{3}}$ are the soft scalar mass for $H_{d}, Q_{3}$ and $U_{3}$, respectively, $\mu B$ is the SUSY breaking mass $\left(\mu B\right.$-term) between $H_{u}$ and $H_{d}$, and $A_{t}$ is the scalar trilinear coupling ( $A$-term) involving the top squarks. Throughout this paper, we neglect all the Yukawa couplings and the $A$-terms except for ones associated to the top quark supermultiplets, $y_{t}$ and $A_{t}$. Note that we use the same notation for denoting a chiral superfield and its lowest scalar component.

The EW symmetry breaking causes the $Z$-boson mass $M_{Z}=91.2 \mathrm{GeV}$. A minimization condition of the total Higgs potential results in the following relation,

$$
\begin{aligned}
\frac{1}{2} M_{Z}^{2} & =-\mu^{2}\left(M_{Z}\right)-\frac{m_{H u}^{2}\left(M_{Z}\right) \tan ^{2} \beta-m_{H_{d}}^{2}\left(M_{Z}\right)}{\tan ^{2} \beta-1} \\
& \sim-\mu^{2}\left(M_{Z}\right)-m_{H u}^{2}\left(M_{Z}\right),
\end{aligned}
$$

where and hereafter we assume a (moderately) large value of $\tan \beta=\left\langle H_{u}\right\rangle /\left\langle H_{d}\right\rangle$ like $\tan \beta \gtrsim 5$. The radiative correction to $m_{H_{u}}^{2}$ is dominantly given by the contributions from 


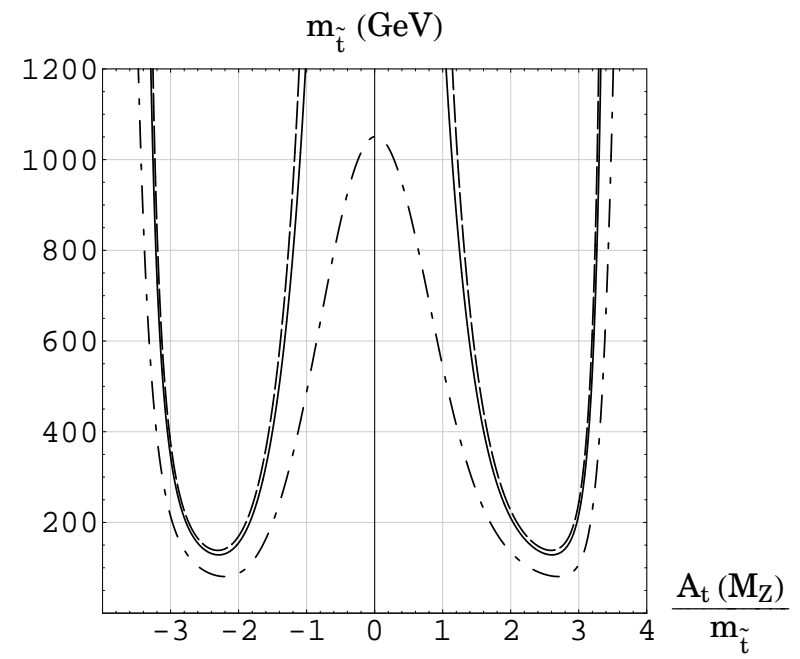

Figure 1: The lower bound on the averaged top squark mass $m_{\tilde{t}}$ for $m_{h} \geq 114.4 \mathrm{GeV}$ (solid line) as well as $m_{h} \geq 110 \mathrm{GeV}$ (dot-dashed line) and $m_{h} \geq 115 \mathrm{GeV}$ (dashed line). The other parameters are chosen as $m_{t}=164.5 \mathrm{GeV}, \mu=200 \mathrm{GeV}$ and $\tan \beta=10$.

top squarks with mass scale $m_{\tilde{t}}$, which is estimated as

$$
\Delta m_{H_{u}}^{2}\left(M_{Z}\right) \approx-\frac{3 y_{t}^{2}\left(M_{Z}\right)}{4 \pi^{2}} m_{\tilde{t}}^{2} \ln \frac{\Lambda}{m_{\tilde{t}}} .
$$

On the other hand, within the two-loop approximation the lightest Higgs boson mass is constrained by [21]

$$
\begin{aligned}
m_{h}^{2} \leq & M_{z}^{2} \cos ^{2} 2 \beta\left(1-\frac{3 m_{t}^{2}}{8 \pi^{2} v^{2}} \ln \frac{m_{\tilde{t}}^{2}}{m_{t}^{2}}\right) \\
& +\frac{3 m_{t}^{4}}{4 \pi^{2} v^{2}}\left[\ln \frac{m_{\tilde{t}}^{2}}{m_{t}^{2}}+\frac{\tilde{A}_{t}^{2}}{m_{\tilde{t}}^{2}}\left(1-\frac{\tilde{A}_{t}^{2}}{12 m_{\tilde{t}}^{2}}\right)\right. \\
& \left.+\frac{1}{16 \pi^{2}}\left(\frac{3 m_{t}^{2}}{2 v^{2}}-32 \pi \alpha_{3}\right)\left\{\frac{2 \tilde{A}_{t}^{2}}{m_{\tilde{t}}^{2}}\left(1-\frac{\tilde{A}_{t}^{2}}{12 m_{\tilde{t}}^{2}}\right) \ln \frac{m_{\tilde{t}}^{2}}{m_{t}^{2}}+\left(\ln \frac{m_{\tilde{t}}^{2}}{m_{t}^{2}}\right)^{2}\right\}\right]
\end{aligned}
$$

where $\tilde{A}_{t}=A_{t}\left(M_{Z}\right)-\mu \cot \beta \approx A_{t}\left(M_{Z}\right)$ and $m_{\tilde{t}}$ is the averaged top squark mass,

$$
m_{\tilde{t}}^{2}=\sqrt{m_{Q_{3}}^{2}\left(M_{Z}\right) m_{U_{3}}^{2}\left(M_{Z}\right)}
$$

The strong gauge coupling $g_{3}$, the vacuum value of the lightest Higgs field $v$, and the running top quark mass $m_{t}$ at the $M_{Z}$ scale are given by $\alpha_{3}\left(M_{Z}\right)=g_{3}^{2} / 4 \pi \approx 0.12$, $v=173.7 \mathrm{GeV}$, and $m_{t}=164.5 \mathrm{GeV}$, respectively.

From the two-loop expression (3) and the observed lower bound by the LEP experiment $m_{h}^{2} \geq 114.4 \mathrm{GeV}$, we can estimate the allowed lowest value of the top squark mass which 
is shown in Fig. 1. From this figure it is apparent that a relatively large $A$-term at the EW scale

$$
\left|A_{t}\left(M_{Z}\right) / m_{\tilde{t}}\right| \gtrsim O(1)
$$

is favorable for the Higgs boson mass above the LEP bound. Furthermore, for a small value of $\left|A_{t}\left(M_{Z}\right) / m_{\tilde{t}}\right|$, a considerably large top squark mass is required as

$$
\begin{aligned}
& m_{\tilde{t}} \gtrsim 500 \mathrm{GeV}, \quad \text { for }\left|A_{t}\left(M_{Z}\right) / m_{\tilde{t}}\right| \lesssim 1.5, \\
& m_{\tilde{t}} \gtrsim 1000 \mathrm{GeV}, \quad \text { for }\left|A_{t}\left(M_{Z}\right) / m_{\tilde{t}}\right| \lesssim 1.0 .
\end{aligned}
$$

This large top squark mass causes $m_{H u}^{2}\left(M_{Z}\right)$ in Eq. (11) to be much larger than $O\left(M_{Z}^{2}\right)$, because of the one-loop effect (2) with a large logarithm. Thus, $\mu^{2}$ must be fine-tuned in order to obtain the successful EW breaking with $M_{Z} \sim 91.2 \mathrm{GeV}$. This is the so-called little hierarchy problem.

The expressions (11), (2) and (3) are all written in terms of the low energy values of parameters such as $m_{H_{u}}^{2}\left(M_{Z}\right)$ and $m_{\tilde{t}}^{2}$. We express the soft parameters at the EW scale in terms of ones at the GUT scale [22], by integrating the one-loop renormalization group equations [1. For example, the gaugino masses at the EW scale are written in terms of themselves at the GUT scale as

$$
\begin{aligned}
& M_{1}\left(M_{z}\right)=0.41 M_{1}, \\
& M_{2}\left(M_{z}\right)=0.82 M_{2}, \\
& M_{3}\left(M_{z}\right)=2.91 M_{3} .
\end{aligned}
$$

On the other hand, the scalar masses $m_{H_{u}}, m_{Q_{3}}, m_{U_{3}}$ and $A_{t}$ at the EW scale are given by

$$
\begin{aligned}
-2 m_{H u}^{2}\left(M_{z}\right)= & 5.45 M_{3}^{2}+0.0677 M_{3} M_{1}-0.00975 M_{1}^{2} \\
& +0.470 M_{2} M_{3}+0.0135 M_{1} M_{2}-0.433 M_{2}^{2} \\
& +0.773 A_{t} M_{3}+0.168 A_{t} M_{2}+0.0271 A_{t} M_{1} \\
& +0.214 A_{t}^{2}-1.31 m_{H u}^{2}+0.690 m_{Q_{3}}^{2}+0.690 m_{U_{3}}^{2}, \\
m_{Q_{3}}^{2}\left(M_{Z}\right)= & 5.76 M_{3}^{2}-0.0113 M_{1} M_{3}-0.00679 M_{1}^{2} \\
& -0.0782 M_{2} M_{3}-0.00225 M_{1} M_{2}+0.400 M_{2}^{2} \\
& -0.129 A_{t} M_{3}+0.0281 A_{t} M_{2}+0.00451 A_{t} M_{1} \\
& -0.0357 A_{t}^{2}-0.115 m_{H_{u}}^{2}+0.885 m_{Q_{3}}^{2}-0.115 m_{U_{3}}^{2}, \\
m_{U_{3}}^{2}\left(M_{Z}\right)= & 4.85 M_{3}^{2}-0.0226 M_{1} M_{3}+0.0453 M_{1}^{2} \\
& -0.156 M_{2} M_{3}-0.00451 M_{1} M_{2}-0.183 M_{2}^{2} \\
& -0.258 A_{t} M_{3}+0.0561 A_{t} M_{2}+0.00903 A_{t} M_{1} \\
& -0.0713 A_{t}^{2}-0.230 m_{H_{u}}^{2}-0.230 m_{Q_{3}}^{2}+0.770 m_{U_{3}}^{2}, \\
A_{t}\left(M_{Z}\right)= & 2.16 M_{3}+0.268 M_{2}+0.0340 M_{1}+0.310 A_{t} .
\end{aligned}
$$

Here the soft parameters without an argument in the right-hand side stand for the values at the GUT scale. We impose the boundary conditions $5 \alpha_{1} / 3=\alpha_{2}=\alpha_{3}=1 / 24$ at the 
GUT scale $M_{G U T}=2 \times 10^{-16} \mathrm{GeV}$ and $y_{t}\left(M_{Z}\right)=m_{t} / v$ at $M_{Z}$. The $\mu$-parameter receives a small radiative correction, and is shown to be

$$
\mu^{2}\left(M_{Z}\right)=1.09 \mu^{2}
$$

The large contribution to the Higgs soft mass (2) from top squarks is now translated into the gluino mass squared $M_{3}^{2}$ with the largest coefficient 5.45 in Eq. (77). The mass squared $M_{3}^{2}$ also appears in $m_{Q_{3}}^{2}\left(M_{Z}\right)$ and $m_{U_{3}}^{2}\left(M_{Z}\right)$ in Eqs. (8) and (9), respectively, as dominant terms. From Eqs. (44), (8) and (9), if all the soft parameters take similar values, i.e., $M_{a} \approx m_{i} \approx A_{t}(a=1,2,3)$, we find

$$
m_{\tilde{t}}^{2} \approx 5 M_{3}^{2}
$$

From Eqs. (5) and (12), the lower bound for $M_{3}$ is estimated as

$$
\begin{array}{ll}
M_{3} \gtrsim 220 \mathrm{GeV}, & \text { for }\left|A_{t}\left(M_{Z}\right) / m_{\tilde{t}}\right| \lesssim 1.5, \\
M_{3} \gtrsim 450 \mathrm{GeV}, & \text { for }\left|A_{t}\left(M_{Z}\right) / m_{\tilde{t}}\right| \lesssim 1.0,
\end{array}
$$

in order to satisfy the Higgs mass bound (3). Thus $M_{3}^{2}$ term with the large coefficient in Eq. (7) and then in Eq. (11) is much larger than $M_{Z}^{2}$. The other terms such as $\mu$ in the right-hand side of Eq. (11) must cancel this large contribution with a good accuracy in order to yield the correct $Z$-boson mass.

From Eq. (7), we also find that this fine-tuning of $\mu$ becomes more severe if we have non-vanishing positive values of $m_{Q_{3}}^{2}$ and $m_{U_{3}}^{2}$ at the GUT scale. 1 Then, as far as the little hierarchy problem is concerned, it is better that the model has vanishing top squark soft masses at the GUT scale,

$$
m_{Q_{3}}^{2}=m_{U_{3}}^{2}=0,
$$

and we adopt this condition in the following analysis.

On the other hand, the Higgs soft mass squared at the GUT scale, $m_{H_{u}}^{2}$, appears in Eq. (7) with a positive coefficient of $O(1)$ and then negative in Eq. (11). Thus, $m_{H_{u}}^{2} \sim$ $O\left(M_{3}^{2}\right)$ can reduce the fine-tuning. We can approximately 'renormalize' this contribution into the $\mu$-parameter effectively of Eqs. (11) and (11), i.e.,

$$
-1.09 \mu^{2} \longrightarrow-1.09 \mu^{2}-0.66 m_{H_{u}}^{2}
$$

in the following discussion of the fine-tuning, because the $m_{H_{u}}^{2}$-terms are negligible in Eqs. (8) and (9) due to the suppressed coefficients of $O(0.1)$. We can easily separate this effect from the effective $\mu$-parameter, if necessary. Then, first we just set

$$
m_{H_{u}}^{2}=0
$$

in the expressions, and consider the $\mu$-term is the effective one when we evaluate an effect due to a non-vanishing value of $m_{H_{u}}^{2}$ at the GUT scale.

\footnotetext{
${ }^{1}$ We can think of introducing tachyonic squarks at the GUT scale, i.e., $m_{Q_{3}}^{2}, m_{U_{3}}^{2}<0$, which can reduce the fine-tuning as is also indicated from Eq. (7). Such possibility has been studied in Ref. [14. In this paper, we study the fine-tuning problem without the tachyonic boundary conditions at the GUT scale.
} 
Based on these arguments, we focus on the contributions from $M_{a}, A_{t}$ and $\mu$ in Eqs. (7), (8) and (9) in the following analysis. Then, we introduce fine-tuning parameters,

$$
\Delta_{X}=\frac{1}{2} \frac{X}{M_{Z}^{2}} \frac{\partial M_{Z}^{2}}{\partial X}, \quad\left(X=\mu, M_{1}, M_{2}, M_{3}, A_{t}\right)
$$

We can easily check that these parameters satisfy the relation,

$$
\sum_{X} \Delta_{X}=1
$$

and then $\Delta_{X} \sim O(1)$ implies that the $Z$-boson mass is insensitive to the parameter $X$ (at the GUT scale). The degree of fine-tuning for the parameter $X$ can be considered as $100 / \Delta_{X}$ percent.

\section{Reducing fine-tuning in bottom-up approach}

In this section, we examine the fine-tuning problem in a bottom-up approach, where all the soft parameters are regarded as independent ones to each other in their origins. For instance, the situation that each gauge kinetic function depends on different (independent) messenger fields may result in the independent gaugino masses at the messenger scale.

In this case, the degree of fine-tuning in the model can be evaluated by the largest one $\Delta_{X}$ among all the fine-tuning parameters defined in Eq. (17) and written explicitly as

$$
\begin{aligned}
\Delta_{M_{1}} & =-0.00975 \hat{M}_{1}^{2}+\left(0.0339 \hat{M}_{3}+0.00675 \hat{M}_{2}+0.0136 \hat{A}_{t}\right) \hat{M}_{1}, \\
\Delta_{M_{2}} & =-0.433 \hat{M}_{2}^{2}+\left(0.235 \hat{M}_{3}+0.00675 \hat{M}_{1}+0.0840 \hat{A}_{t}\right) \hat{M}_{2}, \\
\Delta_{M_{3}} & =5.45 \hat{M}_{3}^{2}+\left(0.0339 \hat{M}_{1}+0.235 \hat{M}_{2}+0.387 \hat{A}_{t}\right) \hat{M}_{3}, \\
\Delta_{A_{t}} & =0.214 \hat{A}_{t}^{2}+\left(0.387 \hat{M}_{3}+0.0840 \hat{M}_{2}+0.0134 \hat{M}_{1}\right) \hat{A}_{t},
\end{aligned}
$$

and

$$
\Delta_{\mu}=-1.09 \hat{\mu}^{2}
$$

where $\hat{M}_{a}=M_{a} / M_{Z}, \hat{A}_{t}=A_{t} / M_{Z}$ and $\hat{\mu}=\mu / M_{Z}$. When we require $\left|\Delta_{\mu}\right| \lesssim 10$, allowed values of $|\mu|$ are $|\mu| \lesssim 280 \mathrm{GeV}$.

We easily find that $\Delta_{M_{3}}$ tends to be the largest among $\Delta_{M_{a}}$ and $\Delta_{A_{t}}$ for the universal gaugino masses $M_{1}=M_{2}=M_{3}=M$ with $A_{t} \sim O\left(M_{a}\right)$. In this case, if we require the fine-tuning for $M_{3}$ to be more than $10 \%$, that is $\Delta_{M_{3}} \approx 6 \hat{M}_{3}{ }^{2} \leq 10$, the gluino mass $M_{3}$ at the GUT scale is restricted as

$$
M \lesssim 120 \mathrm{GeV}, \quad \text { for } \quad M_{1}=M_{2}=M_{3}=M
$$

This does not satisfy the Higgs mass bound (13) for $A_{t}\left(M_{Z}\right) / m_{\tilde{t}} \lesssim 1.5$. Therefore, a larger $A_{t}\left(M_{Z}\right)>1.5 m_{\tilde{t}}$ is inevitable as can be read off from Fig. 1, However, Eq. (10) leads to $A_{t}\left(M_{Z}\right) \sim 2.8 M_{3}$, while Eq. (12) results in $m_{\tilde{t}} \sim 2.2 M_{3}$. Thus, we find $A_{t}\left(M_{Z}\right)>1.5 m_{\tilde{t}}$ 


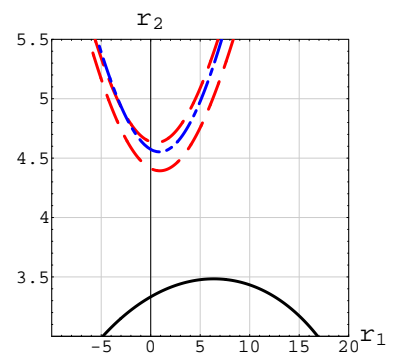

$M=110 \mathrm{GeV}$

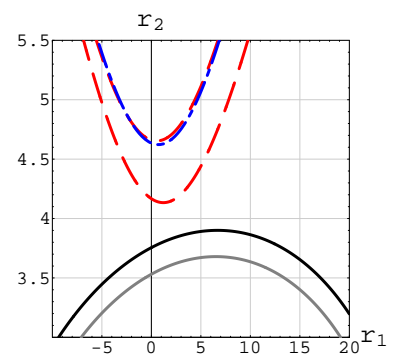

$M=150 \mathrm{GeV}$

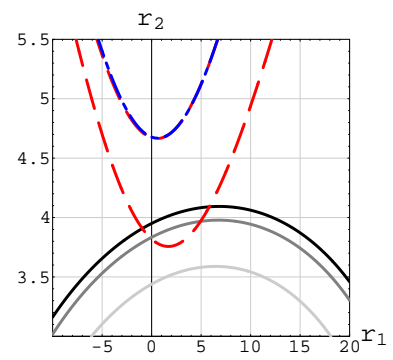

$M=200 \mathrm{GeV}$

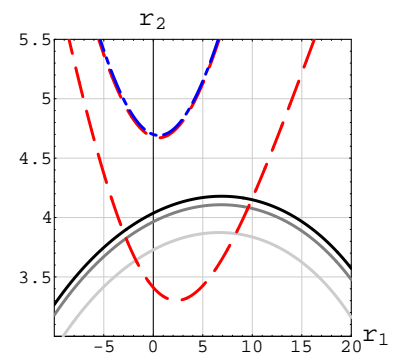

$M=250 \mathrm{GeV}$

Figure 2: Curves for $r_{a}=A_{t} / M_{3}=0$ and $m_{H_{u}}^{2}=m_{Q_{3}, U_{3}}^{2}=0$ determined by constraints from $\Delta_{M}=3.4,5,10$ (solid curves), $m_{h^{0}} \geq 114.4 \mathrm{GeV}$ (between two dashed curves) and $m_{\tilde{t}_{1}} \geq 95.7 \mathrm{GeV}$ (below dot-dashed curves). The parameter $\Delta_{\mu}$ is fixed by the constraint $\Delta_{M}+\Delta_{\mu}=1$. The solid curves are darker for the smaller $\Delta_{M}$.

is impossible. On the other hand, a large value of $M_{3}$ like $M_{3}=220$ or $450 \mathrm{GeV}$ in Eq. (13) leads to a large value of $\Delta_{M_{3}}$ like $\Delta_{M_{3}}=30$ or 130 . For the latter case, we need fine-tuning less than $1 \%$.

The above argument for $M_{1}=M_{2}=M_{3}$ with $A_{t} \sim O\left(M_{a}\right)$ shows that only the possibility to reduce the fine-tuning associated to $M_{3}$ keeping $A_{t} \lesssim O\left(M_{a}\right)$ is a departure from the universal gaugino mass condition at the GUT scale.

Here we denote ratios of gaugino masses and $A_{t}$ by $r_{1}, r_{2}$ and $r_{a}$ as

$$
\left(M_{1}, M_{2}, M_{3}\right)=\left(r_{1}, r_{2}, 1\right) M, \quad A_{t}=r_{a} M,
$$

where $M$ corresponds to the overall magnitude of soft SUSY breaking parameters. Note that we consider ratios, $r_{1}, r_{2}$ and $r_{a}$ are free parameters independent of $M$ in this section. Let us define $\Delta_{M}$ as

$$
\Delta_{M}=\sum_{a=1}^{3} \Delta_{M_{a}}+\Delta_{A_{t}} .
$$

Since $\Delta_{\mu}=1-\Delta_{M}$, we are required to obtain $\Delta_{M} \lesssim O(10)$ in order to avoid fine-tuning of $\Delta_{\mu}$, although this condition is not sufficient and small values $\Delta_{X}$ for $X=M_{1}, M_{2}, M_{3}$ and $A_{t}$ are also required. In $\Delta_{M}$, the dominant contribution is due to $\hat{M}_{3}$ as obvious from Eq. (19). The next important contribution would come from $\hat{M}_{2}$, because of its sign in $\Delta_{M_{2}}$. Indeed, we would obtain $\Delta_{M} \approx 0$ for $r_{2} \approx 4$ when $\hat{M}_{1}=A_{t}=0$. On the other hand, the $\hat{M}_{1}$-dependence of $\Delta_{M}$ would be small, because its coefficient is small. This naive estimation suggests that the parameter region around $r_{2} \sim 4$ would be favorable, while a larger region for $r_{1}$ would be favorable.

As $r_{2}$ increases, $m_{H_{u}}^{2}\left(M_{Z}\right)$ increases and $m_{U_{3}}^{2}\left(M_{Z}\right)$ decreases. For instance, in the extremal case $r_{2} \rightarrow \infty$, the successful electroweak symmetry breaking would not be realized, but the color symmetry would break radiatively. Thus, the parameter $r_{2}$ as well as others is constrained by experimental bounds of the stop mass and $\mu$ and the successful realization of electroweak symmetry breaking.

Figs. 2, 3 and 4 show the contours of $\Delta_{M}=3.4,5,10$ in $\left(r_{1}, r_{2}\right)$-plane for $M=$ $110,150,200$ and $250 \mathrm{GeV}$ in the case of $r_{a}=0,1,2$, respectively. The darkest and 


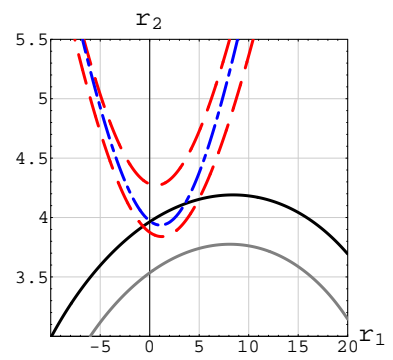

$M=110 \mathrm{GeV}$

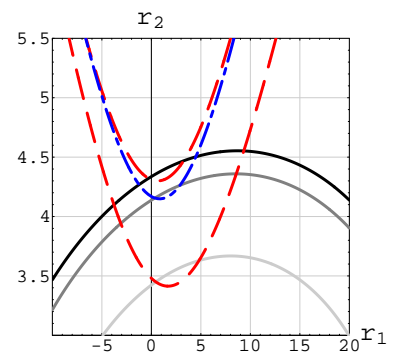

$M=150 \mathrm{GeV}$

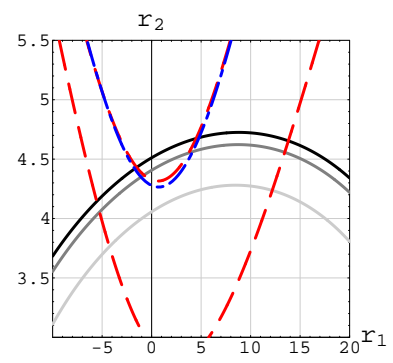

$M=200 \mathrm{GeV}$

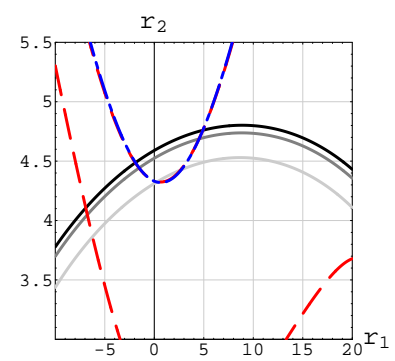

$M=250 \mathrm{GeV}$

Figure 3: The same curves as Fig. 2 but with $r_{a}=A_{t} / M_{3}=1$.

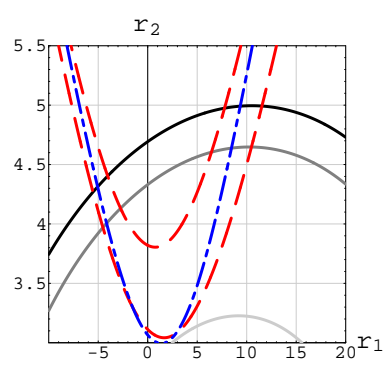

$M=110 \mathrm{GeV}$

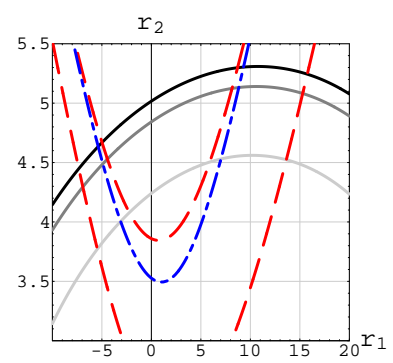

$M=150 \mathrm{GeV}$

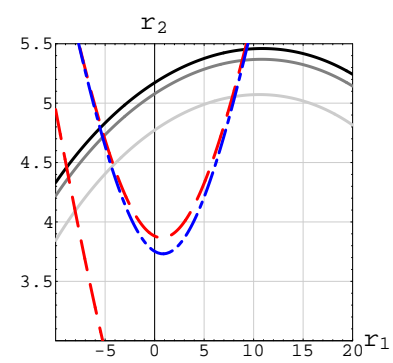

$M=200 \mathrm{GeV}$

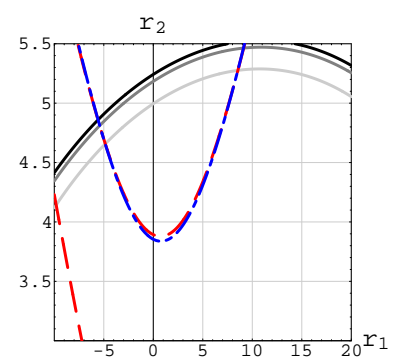

$M=250 \mathrm{GeV}$

Figure 4: The same curves as Fig. 2 but with $r_{a}=A_{t} / M_{3}=2$.

darker solid lines correspond to $\Delta_{M}=3.4$ and 5 , respectively, while the less dark line corresponds to $\Delta_{M}=10$. Above the line corresponding to $\Delta_{M}=3.4$, we can not realize the successful electroweak symmetry breaking when $m_{H_{u}}=0$ and $\left|\mu\left(M_{Z}\right)\right| \geq 94 \mathrm{GeV}$, which corresponds to the experimental bound of chargino mass.

In these figures, we also show the regions satisfying the current Higgs and top squark mass bounds [23], $m_{h} \geq 114.4 \mathrm{GeV}$ and $m_{\tilde{t}_{1}} \geq 95.7 \mathrm{GeV}$, respectively, where $m_{\tilde{t}_{1}}^{2}\left(m_{\tilde{t}_{2}}^{2}\right)$ is the smaller (larger) eigenvalue of the top squark mass-square matrix

$$
M_{\tilde{t}}^{2}=\left(\begin{array}{cc}
m_{Q_{3}}^{2}\left(M_{Z}\right)+m_{t}^{2}+\delta_{Q} & m_{t} \tilde{A}_{t} \\
m_{t} \tilde{A}_{t} & m_{U_{3}}^{2}\left(M_{Z}\right)+m_{t}^{2}+\delta_{U}
\end{array}\right),
$$

with $\delta_{Q}=\left(\frac{1}{2}-\frac{2}{3} \sin ^{2} \theta_{W}\right) \cos (2 \beta) M_{Z}^{2}, \delta_{U}=\frac{2}{3} \sin ^{2} \theta_{W} \cos (2 \beta) M_{Z}^{2}$ and $\sin ^{2} \theta_{W}=1-$ $M_{W}^{2} / M_{Z}^{2}$. We obtain $m_{h} \geq 114.4 \mathrm{GeV}$ between two dashed lines, while we obtain $m_{\tilde{t}_{1}} \geq$ 95.7 GeV below the dot-dashed line, which is close to the upper dashed line in several cases. Figs. 5, 6] show the same contours as Figs. 2, 3] and 4 in $\left(r_{a}, r_{2}\right)$-plane for $M=110,150,200$ and $250 \mathrm{GeV}$ in the case of $r_{1}=2,7.13$, respectively. The ratio $r_{1}=7.13$ is a solution of $M_{1}\left(M_{Z}\right)=M_{3}\left(M_{Z}\right)$, i.e., the unification of the bino and the gluino mass at the EW scale.

From Figs. 2, 3, 4, 5, and 6, we find that the Higgs mass bound as well as the top squark one is satisfied within $\left|\Delta_{M}\right| \leq 10$ for the ratios $r_{1}, r_{2}$ and $r_{a}$ inside the region,

$$
-10 \lesssim r_{1} \lesssim 15, \quad 3.5 \lesssim r_{2} \lesssim 5.5, \quad 0 \lesssim r_{a} \lesssim 2,
$$

when the SUSY breaking scale $M$ varies from $110 \mathrm{GeV}$ to $200 \mathrm{GeV}$. Within this region, 


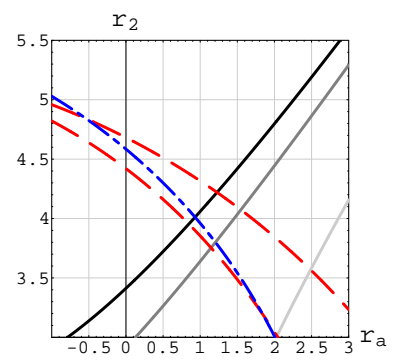

$M=110 \mathrm{GeV}$

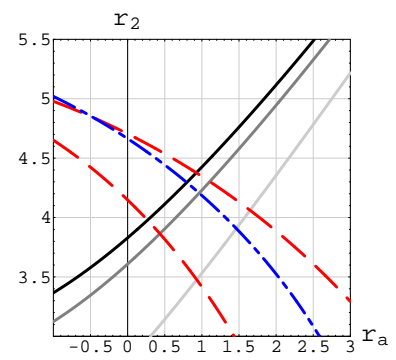

$M=150 \mathrm{GeV}$

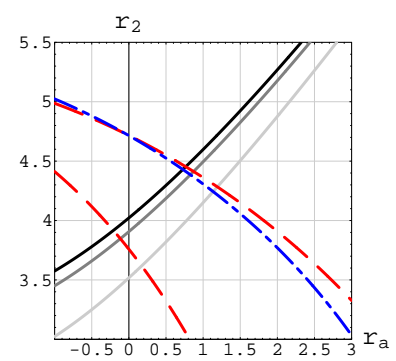

$M=200 \mathrm{GeV}$

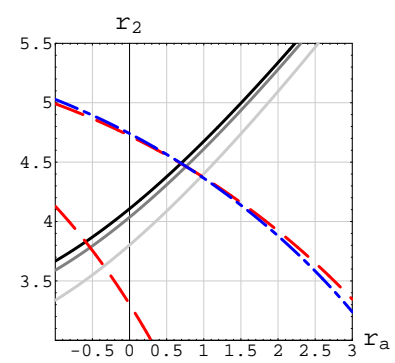

$M=250 \mathrm{GeV}$

Figure 5: Curves for $r_{1}=M_{1} / M_{3}=2$ and $m_{H_{u}}^{2}=m_{Q_{3}, U_{3}}^{2}=0$ determined by constraints from $\Delta_{M}=3.4,5,10$ (solid curves), $m_{h^{0}} \geq 114.4 \mathrm{GeV}$ (between two dashed curves) and $m_{\tilde{t}_{1}} \geq 95.7 \mathrm{GeV}$ (below dot-dashed curves). The parameter $\Delta_{\mu}$ is fixed by the constraint $\Delta_{M}+\Delta_{\mu}=1$. The solid curves are more dark for the smaller $\Delta_{M}$.

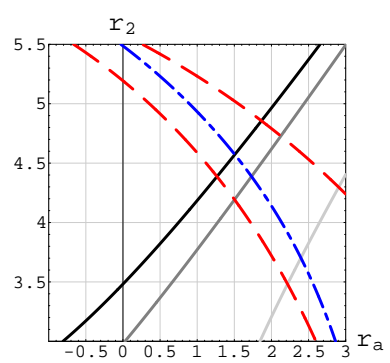

$M=110 \mathrm{GeV}$

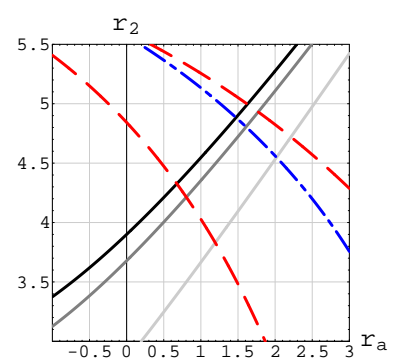

$M=150 \mathrm{GeV}$

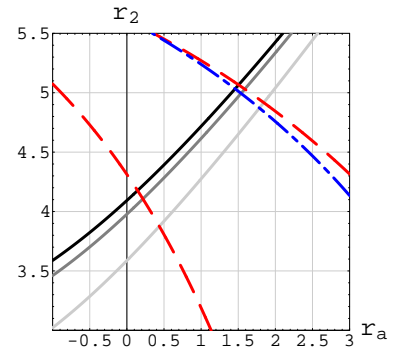

$M=200 \mathrm{GeV}$

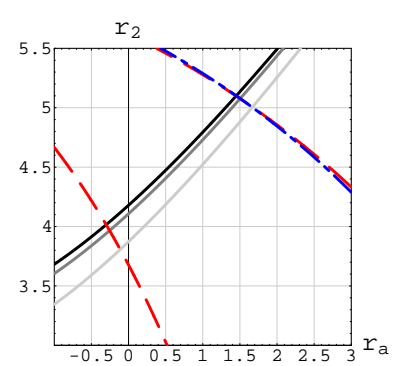

$M=250 \mathrm{GeV}$

Figure 6: The same curves as Fig. 5 but with $r_{1}\left(M_{Z}\right)=M_{1}\left(M_{Z}\right) / M_{3}\left(M_{Z}\right)=1\left(r_{1}=\right.$ 7.13).

the fine-tuning parameter $\Delta_{M_{3}}$ (contained in $\Delta_{M}$ ) given by Eq. (19) is estimated as

$$
5.5\left(M / M_{Z}\right)^{2} \lesssim \Delta_{M_{3}} \lesssim 8\left(M / M_{Z}\right)^{2} .
$$

Thus, in order the fine-tuning associated to $M_{3}$ to be more than $10 \%$, the SUSY breaking scale is restricted by

$$
M \lesssim 110-120 \mathrm{GeV} .
$$

In Fig. 2 with $r_{a}=0$, we find that there is no allowed region for $M \leq 150 \mathrm{GeV}$. Then, from Eq. (25), we conclude that the non-vanishing $A$-term at the GUT scale, $r_{a} \neq 0$, is required for reducing the fine-tuning above $10 \%$ order.

In Table 1, we show some mass spectra for $M=110 \mathrm{GeV}$ (as well as for $M=200 \mathrm{GeV}$ which will be explained later) at some typical points of $\left(r_{1}, r_{2}, r_{a}\right)$ which lead to $\Delta_{M} \sim 5$, i.e., about $20 \%$ tuning in terms of $M$. For $M=110 \mathrm{GeV}$ which is the marginal value of the condition (25)), we find $\Delta_{M_{3}} \sim 10$ and $A_{t}\left(M_{Z}\right) / m_{\tilde{t}} \sim 2$ are realized for $r_{a}=1$ and $r_{a}=2$. These two are distinguished by the masses of the bino and the lighter top squark at the $M_{Z}$ scale. The wino mass is similar to the gluino mass for $r_{a}=1$, and is larger than it for $r_{a}=2$ at the $M_{Z}$ scale. This is because the larger value of $r_{2}$ is preferred for the larger value of $r_{a}$ in Fig. 2 - Fig. 6. 


\begin{tabular}{|cc||c|c||c|c|c|}
\hline$M$ & $(\mathrm{GeV})$ & 110 & 110 & 200 & 200 & 200 \\
\hline \hline$r_{a}$ & 1 & 2 & 0 & 1 & 2 \\
\hline$\left(r_{1}, r_{2}\right)$ & & $(3,4.0)$ & $(10,4.8)$ & $(2,3.9)$ & $(5,4.6)$ & $(10,5.4)$ \\
\hline$\Delta_{M}$ & 3.8 & 4.3 & 5.1 & 4.7 & 4.4 \\
\hline$\Delta_{M_{3}}$ & 10.1 & 11.3 & 31.2 & 34.4 & 38.0 \\
\hline$A_{t}\left(M_{Z}\right) / m_{\tilde{t}}$ & 2.0 & 1.9 & 1.6 & 2.4 & 2.2 \\
\hline$M_{3}\left(M_{Z}\right)$ & $(\mathrm{GeV})$ & 321 & 321 & 583 & 583 & 583 \\
\hline$M_{2}\left(M_{Z}\right)$ & $(\mathrm{GeV})$ & 361 & 433 & 640 & 755 & 886 \\
\hline$M_{1}\left(M_{Z}\right)$ & $(\mathrm{GeV})$ & 135 & 450 & 164 & 409 & 818 \\
\hline$\mu\left(M_{Z}\right)$ & $(\mathrm{GeV})$ & 108 & 117 & 130 & 125 & 120 \\
\hline$m_{\tilde{t}_{2}}$ & $(\mathrm{GeV})$ & 436 & 468 & 714 & 764 & 820 \\
\hline$m_{\tilde{t}_{1}}(\mathrm{GeV})$ & 202 & 131 & 247 & 133 & 186 \\
\hline$m_{h, \max }(\mathrm{GeV})$ & 115 & 115 & 115 & 120 & 120 \\
\hline \multicolumn{2}{r}{} & & & & & \\
\hline
\end{tabular}

Table 1: The mass spectra for $M=110$ and $200 \mathrm{GeV}$ at some typical points of $\left(r_{1}, r_{2}, r_{a}\right)$ which lead to $\Delta_{M} \sim 5$ (20\% tuning).

Finally in this section, we summarize the discussions above. If all the soft parameters (as well as the $\mu$-term) at the GUT scale are independent to each other in their origins, the degree of fine-tuning in the model is almost determined by $\Delta_{M_{3}}$. A numerical evaluation indicates that only the possibility for relaxing the fine-tuning above $10 \%$ order $\left(\Delta_{M_{3}} \lesssim 10\right)$ resides in the case of i) non-universal gaugino masses with the ratio inside the region (24), ii) a non-vanishing $A$-term at the GUT scale, $A_{t}>0$, and iii) a considerably low SUSY breaking scale (25).

\section{Fine-tuning with fixed ratios}

It is reasonable enough to consider the situation that some or all of the soft SUSY breaking parameters share a common mass scale $M$, and the ratios between them are determined by some dimension less constants and/or geometrical numbers such as beta function coefficients, modular weights, and so on. Indeed, ratios of soft SUSY breaking parameters are fixed as certain values in each model, e.g. in moduli mediation, anomaly mediation, gauge messenger model and so on. In this case, we do not need to worry about all of the fine-tuning parameters (19), and the degree of fine-tuning in the model is represented by only $\Delta_{M}$.

In this section, we reexamine the discussions in the previous section, by assuming that the ratio $r_{1}, r_{2}$ and $r_{a}$ in Eq. (22) is fixed to some numbers by the UV theory. In this case, the remaining fine-tuning parameters are $\Delta_{M}$ and $\Delta_{\mu}$ given by Eqs. (23) and (20), respectively. In other words, we worry about the sensitivity of the $Z$-boson mass to only the common SUSY breaking scale $M$ and the SUSY mass scale $\mu$. The $\Delta_{M_{3}}$ is a meaningless parameter in this sense, and thus the SUSY breaking scale $M$ is released from the previous upper bounds (25) or (21). The numerical results in Fig. 2-Fig. 6 show that $M \sim 200 \mathrm{GeV}$ possesses the widest allowed region of the ratios $r_{1}, r_{2}$ and $r_{a}$. This 


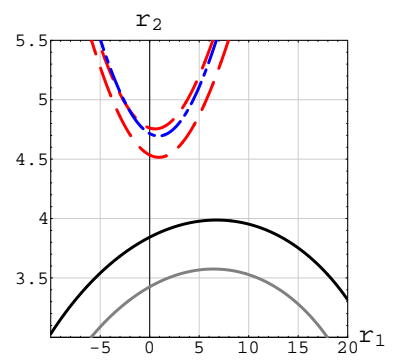

$M=110 \mathrm{GeV}$

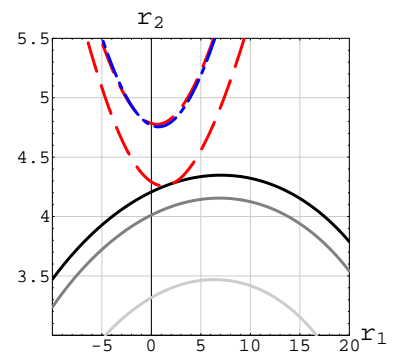

$M=150 \mathrm{GeV}$

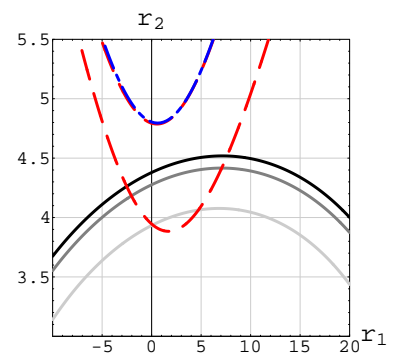

$M=200 \mathrm{GeV}$

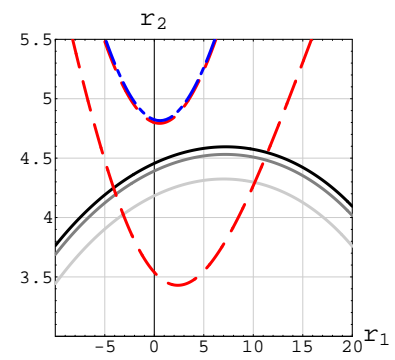

$M=250 \mathrm{GeV}$

Figure 7: The same curves as Fig. 2 but with $m_{H_{u}}^{2}=-M^{2}$.

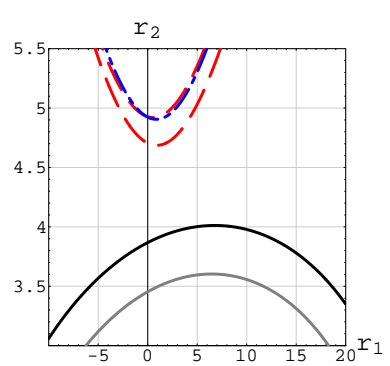

$M=110 \mathrm{GeV}$

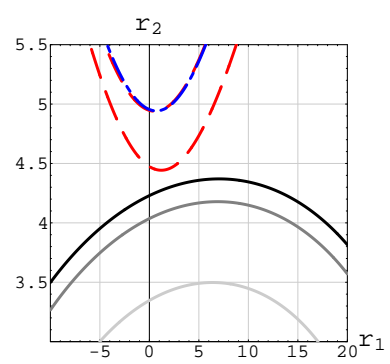

$M=150 \mathrm{GeV}$

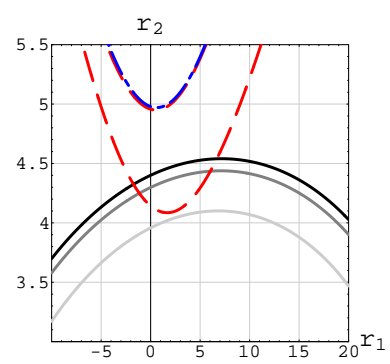

$M=200 \mathrm{GeV}$

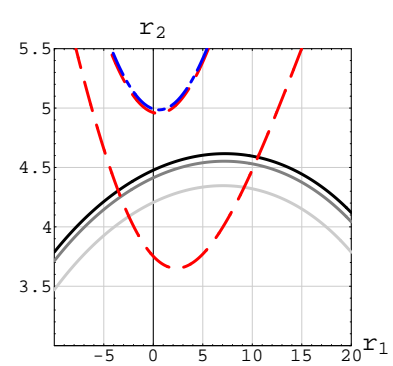

$M=250 \mathrm{GeV}$

Figure 8: The same curves as Fig. 2 but with $m_{Q_{3}, U_{3}}^{2}=M^{2}$.

is because the smaller $M$ results in a smaller allowed region for the Higgs and the top squark mass bounds, while the region where $\Delta_{M} \leq 10$ becomes narrower for the larger $M$. These opposite tendencies are balanced at $M \sim 200 \mathrm{GeV}$.

It is remarkable that the fine-tuning can be completely improved in this case. For some values of $r_{1}, r_{2}$ and $r_{a}$ inside (24), the fine-tuning parameter $\Delta_{M}$ can be of $O(1)$, and then $\Delta_{\mu}$ is also of $O(1)$ from Eq. (18). Note that $\Delta_{M_{3}}$ is still large $\Delta_{M_{3}} \gg 1$ for $M>120 \mathrm{GeV}$. The point is, however, now the fine-tuning parameter is not $\Delta_{M_{3}}$ but the total sum $\Delta_{M} \sim O(1)$, where a cancellation occurs between $\Delta_{M_{3}}$ and $\Delta_{M_{1,2}, A_{t}}$.

At any rate, irrespective of whether we worry about the fine-tuning parameter $\Delta_{M_{3}}$ or not, we can obtain $\Delta_{M} \sim O(1)$ in the region (24) and then $\Delta_{\mu} \sim O(1)$. This implies the $Z$-boson mass is insensitive to not only the SUSY breaking scale $M$ but also the SUSY mass scale $\mu$. The small $\Delta_{\mu}$ corresponds to the small value of $\mu$ itself. It can be even the marginal value to the current chargino mass bound. The small Higgsino mass is a general consequence of reduced fine-tuning associated to the $\mu$-parameter.

What the favored region of the ratios (24) indicates? First, this region is mostly close to the minimum of $m_{\tilde{t}}$ in terms of $A_{t}\left(M_{Z}\right) / m_{\tilde{t}}$ in Fig. 1, that is the large top squark mixing case [11, 14]. Second, the favored ratios between gaugino masses may be explained as follows. The region (24) corresponds to

$$
-1.4 \lesssim r_{1}\left(M_{Z}\right) \lesssim 2.1, \quad 1.0 \lesssim r_{2}\left(M_{Z}\right) \lesssim 1.4,
$$

where

$$
r_{1}\left(M_{Z}\right)=M_{1}\left(M_{Z}\right) / M_{3}\left(M_{Z}\right)=0.14 r_{1},
$$




$$
r_{2}\left(M_{Z}\right)=M_{2}\left(M_{Z}\right) / M_{3}\left(M_{Z}\right)=0.28 r_{2},
$$

are the gaugino mass ratios at the $Z$-boson mass scale. Favorable region of $r_{2}\left(M_{Z}\right)$ is rather wide, e.g. $\Delta r_{2} / r_{2}\left(M_{Z}\right)=O(0.1)$, where $r_{2}\left(M_{Z}\right)=1.2$ and $\Delta r_{2}\left(M_{Z}\right)=0.2$. We have much wider favorable region for $r_{1}\left(M_{Z}\right)$. That is important from the viewpoint of model building, because that allows $10 \%$ uncertainty for an explicit model. The ratio $r_{2}\left(M_{Z}\right) \approx 1$ indicates the unification of the wino and the gluino masses at the EW scale. Then the reduced fine-tuning can be explained in the terminology of the so-called mirage mediation [9] of SUSY breaking. The mirage unification of the gaugino masses at the EW scale [9, 10] implies that the large logarithmic correction (2) to the $m_{H_{u}}^{2}$ is completely canceled at the EW scale due to the special boundary conditions at the GUT scale as a consequence of the mixed modulus-anomaly mediation 2 . The range of the ratios (24) includes this type of boundary conditions as the central values.

However, from (26) we find that, in order to reduce the fine-tuning, it is not necessary that all the gaugino masses are unified at the EW scale as in the mirage mediation models. The important one is the wino/gluino mass ratio, and we have a wider choice for the value of bino/gluino mass ratio as long as the fine-tuning is concerned. Inversely, the relaxed fine-tuning may predict the unification of the wino and gluino masses at the EW scale, but not the bino-gluino unification.

In Table 1, the mass spectra for $M=200 \mathrm{GeV}$ are shown at some typical points of $\left(r_{1}, r_{2}, r_{a}\right)$ which lead to $\Delta_{M} \sim 5$, i.e., about $20 \%$ tuning in terms of $M$. The vanishing $A_{t}$ at the GUT scale $r_{a}=0$ is possible for $M=200 \mathrm{GeV}$ as well as $r_{a}=1,2$. In the case of $r_{a}=0$, the large $A_{t}\left(M_{Z}\right) / m_{\tilde{t}} \sim O(1)$ at the $Z$-boson mass scale is generated radiatively. The three cases $r_{a}=0,1,2$ are most likely distinguished by the mass of the bino at the $M_{Z}$ scale. This is due to the fact that the larger $r_{a}$ prefers the larger $r_{1}$ for $\Delta_{M} \leq 5$ as can be seen by comparing Fig. 2 - Fig. 4. The wino mass is similar to the gluino mass for $r_{a}=0$, and is larger than it for $r_{a}=1,2$ at the $M_{Z}$ scale. Because we are now taking such a stance that the gaugino masses are not independent in their origins, the value of $\Delta_{M_{3}}$ is meaningless, although it is shown in Table 1 for the purpose of reference.

So far, we have considered the case with vanishing soft scalar masses, $m_{H_{u}}=m_{Q_{3}}=$ $m_{U_{3}}=0$. Here we comment on effects due to non-vanishing soft scalar masses. First, let us evaluate effects due to non-vanishing value of the Higgs soft scalar mass $m_{H_{u}}$. Its effect on stop masses is small. That implies that the lightest Higgs mass $m_{h}$ and stop masses $m_{\tilde{t}}$ would not change significantly even when we vary $m_{H_{u}}$ in the region with $\left|m_{H_{u}}^{2}\right| \lesssim O\left(M^{2}\right)$. A significant effect appears only in $m_{H_{u}}^{2}\left(M_{Z}\right)$, and such effect can be understood as 'renormalization' (15). That is, the favorable region with small $\Delta_{M}$ shifts toward the region with larger (smaller) $r_{2}$, when $m_{H_{u}}$ becomes negative (positive). Fig. 7 shows the case with $m_{H_{u}}^{2}=-M^{2}$. Next, we comment on effects due to non-vanishing values of $m_{Q_{3}}$ and $m_{U_{3}}$. Their effects on $\Delta_{M}$ are almost opposite to the above effect of $m_{H_{u}}$, because their signs are opposite in Eq. (7). The small $\Delta_{M}$ region shifts toward the region with larger (smaller) $r_{2}$, when $m_{Q_{3}}=m_{U_{3}}$ becomes positive (negative). Furthermore,

\footnotetext{
${ }^{2}$ In the flux compactification models 24, the mirage unification scale is determined by the modulus/anomaly ratio of SUSY breaking mediation [18, which depends on the dilaton-modulus mixing ratios in the nonperturbative superpotential [25, 27. as well as how we uplift the AdS minimum to dS one $[26,27,28]$.
} 
they also affect on the lightest Higgs mass $m_{h}$ and stop masses $m_{\tilde{t}}$. Then, totally the favorable region shifts slightly when we vary $m_{Q_{3}}=m_{U_{3}}$, but the wideness of favorable region does not change drastically. Fig. 8 shows the case with $m_{Q_{3}, U_{3}}^{2}=M^{2}$.

\section{Conclusions}

We studied the fine-tuning problem between the soft SUSY breaking parameters and the $\mu$-term for the successful electroweak symmetry breaking in the MSSM. The bottomup considerations lead us to the non-universal gaugino masses at the GUT scale as a necessary condition for reducing the fine-tuning above $10 \%$ order, if all the soft parameters are regarded as independent ones to each other in their origins and no tachyonic superparticles are assumed at the GUT scale. In this case, the small gluino mass $M_{3} \lesssim 120$ $\mathrm{GeV}$ and the non-vanishing $A$-terms $A_{t}>0$ at the GUT scale is required from $\Delta_{M_{3}} \lesssim 10$.

On the other hand, if the soft SUSY breaking parameters share a common mass scale $M$ with the fixed ratios by the UV theory, each fine-tuning parameter such as $\Delta_{M_{3}}$ does not make any sense. Only the total one such as $\Delta_{M}=\sum_{a=1}^{3} \Delta_{M_{a}}+\Delta_{A_{t}}$ as well as the SUSY parameter $\Delta_{\mu}$ represents the degree of fine-tuning in the model. In this case, the above upper-bound on $M_{3}$ disappears, and then we find the fine-tuning can be completely improved in some models of non-universal gaugino masses. A numerical evaluation shows that the model with the gluino mass $M_{3} \sim 200 \mathrm{GeV}$ at the GUT scale has the widest allowed range of $r_{1}=M_{1} / M_{3}, r_{2}=M_{2} / M_{3}$ and $r_{a}=A_{t} / M_{3}$. In this case of the least fine-tuning, even the vanishing $A_{t}$ at the GUT scale is possible and a relatively large $A_{t}\left(M_{Z}\right) / m_{\tilde{t}}>1.5$ at the $Z$-boson mass scale is generated radiatively.

In both the above approaches, the non-universal gaugino mass conditions, especially, $M_{2} \approx 4 M_{3}$ at the GUT scale is the key to improve the fine-tuning. This implies the wino and gluino degeneracy at the weak scale. Another implication is a smaller Higgsino mass due to the reduced or eliminated fine-tuning $\Delta_{M} \leq 10$ accompanying $\left|\Delta_{\mu}\right| \leq 10$. The bino mass $M_{1}$ at the GUT scale is less constrained from our discussions of finetuning. This fact implies that the EW mirage-unification model [9, 10], where all the gaugino masses are unified at the EW scale, can be deformed such that only the wino and gluino masses are unified, keeping the absence of fine-tuning. In other words, the $U(1)_{Y}$ gauge kinetic function can have a different origin from the other ones for $S U(3)_{C}$ and $S U(2)_{L}$. It would be important to study model building at high energy scale, extending the low-energy scale mirage [9, 10]. We would study elsewhere explicit construction of such partial mirage model, where only the gluino and wino masses are degenerate around $M_{Z}$. A negative value of $m_{H_{u}}$ makes the favorable region wider, and larger value of $M_{2} / M_{3}$ becomes favorable. On the other hand, when we vary stop masses $m_{Q_{3}, U_{3}}$, the situation does not change drastically.

Our favorable value of $\mu$ is small. For example we have $|\mu| \lesssim 280 \mathrm{GeV}$, when we require $\Delta_{\mu} \lesssim 10$. In addition, the bino mass $M_{1}$ can vary in a quite wide range. This aspect would be interesting from the viewpoint of dark matter candidate.

We have concentrated to the Higgs sector and the electroweak symmetry breaking, to which only gaugino masses, stop masses and Higgs masses are relevant. Other mass parameters are irrelevant to our discussion, that is, they can be more model-dependent. 


\section{Acknowledgement}

The authors would like to thank Haruhiko Terao for useful discussions. H. A. and T. K. are supported in part by the Grand-in-Aid for Scientific Research \#182496, \#17540251, respectively. T. K. is also supported in part by the Grant-in-Aid for the 21st Century COE "The Center for Diversity and Universality in Physics" from the Ministry of Education, Culture, Sports, Science and Technology of Japan.

\section{References}

[1] K. Inoue, A. Kakuto, H. Komatsu and S. Takeshita, Prog. Theor. Phys. 67, 1889 (1982); K. Inoue, A. Kakuto, H. Komatsu and S. Takeshita, Prog. Theor. Phys. 68, 927 (1982) [Erratum-ibid. 70, 330 (1983)]; K. Inoue, A. Kakuto, H. Komatsu and S. Takeshita, Prog. Theor. Phys. 71, 413 (1984); L. E. Ibanez and G. G. Ross, Phys. Lett. B 110, 215 (1982); L. Alvarez-Gaume, M. Claudson and M. B. Wise, Nucl. Phys. B 207, 96 (1982).

[2] Y. Okada, M. Yamaguchi and T. Yanagida, Phys. Lett. B 262, 54 (1991); H. E. Haber and R. Hempfling, Phys. Rev. Lett. 66, 1815 (1991); J. R. Ellis, G. Ridolfi and F. Zwirner, Phys. Lett. B 262, 477 (1991).

[3] R. Barbieri and G. F. Giudice, Nucl. Phys. B 306, 63 (1988); P. H. Chankowski, J. R. Ellis and S. Pokorski, Phys. Lett. B 423, 327 (1998) hep-ph/9712234; P. H. Chankowski, J. R. Ellis, M. Olechowski and S. Pokorski, Nucl. Phys. B 544, 39 (1999) hep-ph/9808275]; G. L. Kane and S. F. King, Phys. Lett. B 451, 113 (1999) hep-ph/9810374]; M. Bastero-Gil, G. L. Kane and S. F. King, Phys. Lett. B 474, 103 (2000) [hep-ph/9910506]; G. L. Kane, J. D. Lykken, B. D. Nelson and L. T. Wang, Phys. Lett. B 551, 146 (2003) [hep-ph/0207168].

[4] A. Brignole, J. A. Casas, J. R. Espinosa and I. Navarro, Nucl. Phys. B 666, 105 (2003) hep-ph/0301121]; J. A. Casas, J. R. Espinosa and I. Hidalgo, JHEP 0401, 008 (2004) [hep-ph/0310137]: JHEP 0411, 057 (2004) hep-ph/0410298].

[5] P. Batra, A. Delgado, D. E. Kaplan and T. M. P. Tait, JHEP 0402, 043 (2004) hep-ph/0309149.

[6] R. Harnik, G. D. Kribs, D. T. Larson and H. Murayama, Phys. Rev. D 70, 015002 (2004) hep-ph/0311349]; S. Chang, C. Kilic and R. Mahbubani, Phys. Rev. D 71, 015003 (2005) |hep-ph/0405267]; A. Delgado and T. M. P. Tait, JHEP 0507, 023 (2005) hep-ph/0504224.

[7] T. Kobayashi and H. Terao, JHEP 0407, 026 (2004) hep-ph/0403298]; T. Kobayashi, H. Nakano and H. Terao, Phys. Rev. D 71, 115009 (2005) [hep-ph/0502006]; T. Kobayashi, H. Terao and A. Tsuchiya, Phys. Rev. D 74, 015002 (2006) hep-ph/0604091. 
[8] A. Birkedal, Z. Chacko and Y. Nomura, Phys. Rev. D 71, 015006 (2005) [hep-ph/0408329]; A. Birkedal, Z. Chacko and M. K. Gaillard, JHEP 0410, 036 (2004) (hep-ph/0404197]; Z. Chacko, Y. Nomura and D. Tucker-Smith, Nucl. Phys. B 725, 207 (2005) [hep-ph/0504095].

[9] K. Choi, K. S. Jeong and K. i. Okumura, JHEP 0509, 039 (2005) hep-ph/0504037.

[10] K. Choi, K. S. Jeong, T. Kobayashi and K. i. Okumura, Phys. Lett. B 633, 355 (2006) [hep-ph/0508029]; hep-ph/0612258.

[11] R. Kitano and Y. Nomura, Phys. Lett. B 631, 58 (2005) hep-ph/0509039.

[12] A. Falkowski, S. Pokorski and M. Schmaltz, Phys. Rev. D 74, 035003 (2006) hep-ph/0604066]; S. Chang, L. J. Hall and N. Weiner, hep-ph/0604076.

[13] G. F. Giudice and R. Rattazzi, Nucl. Phys. B 757, 19 (2006) hep-ph/0606105]; L. M. Carpenter, D. E. Kaplan and E. J. Rhee, hep-ph/0607204; S. G. Kim, N. Maekawa, A. Matsuzaki, K. Sakurai, A. I. Sanda and T. Yoshikawa, Phys. Rev. D 74, 115016 (2006) [hep-ph/0609076]; R. Dermisek, J. F. Gunion and B. McElrath, hep-ph/0612031.

[14] R. Dermisek and H. D. Kim, Phys. Rev. Lett. 96, 211803 (2006) hep-ph/0601036]; K. J. Bae, R. Dermisek, H. D. Kim and I. W. Kim, hep-ph/0702041.

[15] R. Dermisek, H. D. Kim and I. W. Kim, JHEP 0610, 001 (2006) hep-ph/0607169].

[16] A. Brignole, L. E. Ibanez and C. Munoz, Nucl. Phys. B 422, 125 (1994) [Erratumibid. B 436, 747 (1995)] [hep-ph/9308271]; T. Kobayashi, D. Suematsu, K. Yamada and Y. Yamagishi, Phys. Lett. B 348, 402 (1995) [hep-ph/9408322]; L. E. Ibanez, C. Munoz and S. Rigolin, Nucl. Phys. B 553, 43 (1999) [hep-ph/9812397].

[17] L. Randall and R. Sundrum, Nucl. Phys. B 557, 79 (1999) hep-th/9810155); G. F. Giudice, M. A. Luty, H. Murayama and R. Rattazzi, JHEP 9812, 027 (1998) hep-ph/9810442.

[18] K. Choi, A. Falkowski, H. P. Nilles, M. Olechowski and S. Pokorski, JHEP 0411, 076 (2004) hep-th/0411066]; K. Choi, A. Falkowski, H. P. Nilles and M. Olechowski, Nucl. Phys. B 718, 113 (2005) hep-th/0503216.

[19] J. R. Ellis, C. Kounnas and D. V. Nanopoulos, Nucl. Phys. B 247, 373 (1984). J. R. Ellis, K. Enqvist, D. V. Nanopoulos and K. Tamvakis, Phys. Lett. B 155, 381 (1985). M. Drees, Phys. Lett. B 158, 409 (1985). G. Anderson, C. H. Chen, J. F. Gunion, J. D. Lykken, T. Moroi and Y. Yamada, hep-ph/9609457; K. Huitu, Y. Kawamura, T. Kobayashi and K. Puolamaki, Phys. Rev. D 61, 035001 (2000) hep-ph/9903528.

[20] K. Choi and H. P. Nilles, hep-ph/0702146. 
[21] M. Carena, M. Quiros and C. E. M. Wagner, Nucl. Phys. B 461, 407 (1996) [hep-ph/9508343]; M. Carena, H. E. Haber, S. Heinemeyer, W. Hollik, C. E. M. Wagner and G. Weiglein, Nucl. Phys. B 580, 29 (2000) [hep-ph/0001002].

[22] L. E. Ibanez and C. Lopez, Nucl. Phys. B 233, 511 (1984); L. E. Ibanez, C. Lopez and C. Munoz, Nucl. Phys. B 256, 218 (1985); A. Lleyda and C. Munoz, Phys. Lett. B 317, 82 (1993) [hep-ph/9308208].

[23] W. M. Yao et al. [Particle Data Group], J. Phys. G 33, 1 (2006).

[24] S. Kachru, R. Kallosh, A. Linde and S. P. Trivedi, Phys. Rev. D 68, 046005 (2003) [hep-th/0301240].

[25] H. Abe, T. Higaki and T. Kobayashi, Phys. Rev. D 73, 046005 (2006) hep-th/0511160].

[26] O. Lebedev, H. P. Nilles and M. Ratz, Phys. Lett. B 636, 126 (2006) hep-th/0603047.

[27] K. Choi and K. S. Jeong, JHEP 0608, 007 (2006) hep-th/0605108.

[28] E. Dudas, C. Papineau and S. Pokorski, hep-th/0610297; H. Abe, T. Higaki, T. Kobayashi and Y. Omura, Phys. Rev. D 75, 025019 (2007) hep-th/0611024; R. Kallosh and A. Linde, hep-th/0611183; O. Lebedev, V. Lowen, Y. Mambrini, H. P. Nilles and M. Ratz, hep-ph/0612035. 